\section{Comparação entre duas curvas de crescimento para detectar recém-nascidos pequenos para a idade gestacional}

\section{Comparison of two growth curves for detecting small-for-gestational-age newborns}

Brunnella Alcantara Chagas de Freitas 1

Luciana Moreira Lima 2

Maria Elisabeth Moreira Lopes 3

Carla Fernanda Lisboa Valente Carlos 4

Silvia Eloiza Priore 5

Sylvia do Carmo Castro Franceschini 6

1,2 Departamento de Medicina e Enfermagem. Universidade Federal de Viçosa. Avenida P. H. Rolfs, s/n, Campus Universitário. CEP: 36.570-900. Viçosa, MG, Brasil. E-mail: brunnella.freitas@ufv.br

3 Instituto Fernandes Figueira. Fundação Oswaldo Cruz. Rio de Janeiro, RJ, Brasil.

4 Programa de Pós-graduação em Assistência Hospitalar ao Neonato. Fundação Educacional Lucas Machado. Belo Horizonte, MG, Brasil.

5,6 Departamento de Nutrição e Saúde. Universidade Federal de Viçosa. Viçosa, MG, Brasil.

\section{Resumo}

Objetivos: comparar duas curvas de crescimento, Lubchenco e Fenton e Kim, na detecção de recémnascidos (RN) pequenos para idade gestacional (PIG) segundo sexo.

Métodos: estudo transversal de dados de RN, de 01/01/2010 a 31/12/2012 Definiu-se PIG o peso ao nascer $(P N)$ inferior ao percentil 10 para idade gestacional (IG) segundo as curvas Lubchenco e Fenton \& Kim. Mensurou-se a concordancia entre as curvas pelo Método de Kappa para o diagnóstico de PIG segundo sexo e IG. Construíram-se curvas dos percentis 10 de $P N$ e referenciais, por sexo, utilizando função polinomial de terceiro grau. Para comparar e medir associações entre as variáveis sexo, IG e PIG foram utilizados o teste do qui-quadrado de Pearson e regressão logística bivariada, respectivamente. Significância: $p<0,05$.

Resultados: foram incluidos 2364 recém-nascidos no estudo. As curvas Fenton e Kim detectaram mais RN PIG, cuja taxa foi 16,2\%, enquanto as Lubchenco detectaram 3,3\% $(p<0,001 ; k=0,292)$. A discordância foi maior com o aumento da IG. O sexo masculino se associou à maior chance de nascer PIG 1,6 vezes em relação ao feminino $(O R=1,59 ; I C 95 \%=1,28-2,00$; $p<0,001)$.

Conclusões: as curvas Fenton e Kim detectaram mais RN PIG. O nascimento PIG foi mais frequente no sexo masculino. Os achados contribuem para adoção de estratégias visando melhorias no diagnóstico, cuidados e prognóstico.

Palavras-chave Recém-nascido pequeno para idade gestacional, Peso ao nascer 
Introdução

O peso de nascimento é um importante preditor de morbimortalidade perinatal. ${ }^{1}$ As crianças que nascem pequenas para a idade gestacional (PIG), além de estarem sob risco imediato de desenvolver uma série de problemas no período neonatal, ${ }^{2}$ também estão mais sujeitas ao desenvolvimento de doenças crônicas na idade adulta, tais como diabetes, hipertensão e doença coronariana. ${ }^{3}$

Define-se como PIG aquele recém-nascido (RN) com peso ao nascer (PN) inferior ao percentil 10 para sua idade gestacional (IG), de acordo com a curva de referência utilizada. Depois do surgimento das curvas de Lubchenco et al. ${ }^{4} \mathrm{em} 1963$, que ainda são amplamente utilizadas, outros referenciais para avaliar o PN de acordo com a IG têm sido propostos na literatura, como as curvas de Usher e McLean 5 (1969), Alexander et al.6 (1996), Olsen et al.7 (2010) e Fenton e $\mathrm{Kim}^{8}$ (2013) e mais recentemente as curvas do Intergrowth. 9

Apesar de ainda existir na literatura uma certa discordância sobre o ponto de corte no percentil para definição de PIG, o percentil 10 tem sido mais utilizado que o percentil 3. As curvas de Lubchenco et al. 4 utilizam como ponto de corte o percentil $10 \mathrm{e}$ as de Usher e McLean 5 definiram como limite inferior o percentil 3. Em 1996, Alexander et al. 6 desenvolveram uma curva de referência, amplamente utilizada nos EUA até 2010, sendo então substituída pela de Olsen et al. 7 Em 2013, Fenton e Kim 8 realizaram uma metanálise incluindo estudos de seis países desenvolvidos e abrangendo a maior amostra de dados de RN até o momento e desenvolveram novas curvas para avaliar o crescimento dos RN de acordo com a IG.

A escolha de uma curva de referência adequada para cada serviço é importante porque ela determina o ponto de corte para a monitorização de problemas metabólicos que podem comprometer a qualidade de vida como por exemplo a hipoglicemia. Considerando que o uso de diferentes curvas de referência que avaliam a adequação do PN para a IG cria diversas possibilidades de resultados, tornou-se necessário analisar comparativamente este parâmetro que reflete a qualidade do crescimento intrauterino e suas consequências neonatais imediatas. Assim, o presente estudo objetivou comparar duas curvas de crescimento, Lubchenco et al. 4 e Fenton e Kim, ${ }^{8}$ na detecção de recém-nascidos (RN) pequenos para idade gestacional (PIG) segundo sexo.

\section{Métodos}

Trata-se de estudo transversal, a partir de dados de recém-nascidos obtidos no Hospital São Sebastião (HSS), em Viçosa-MG, referência para gestação de alto risco, no período de três anos, entre 01/01/2010 a $31 / 12 / 2012$. Os dados foram coletados a partir do livro de registro de nascimentos do referido hospital, considerando-se uma amostragem de conveniência. Para uma prevalência de RN PIG de 50\% com precisão de $5 \%$ e significância de $95 \%$, seriam necessários os dados de $384 \mathrm{RN}$.

No HSS são realizados todos os partos do município de Viçosa e Região de Saúde (Viçosa, Araponga, Cajuri, Canaã, Paula Cândido, Pedra do Anta, Porto Firme, São Miguel, Teixeiras). O Hospital é referência para gestação de alto risco desde 2009 e, além disso, em convênio com a Universidade Federal de Viçosa (UFV) participa da formação de alunos e médicos residentes desde 2011.

Foram incluídos para análise todos os dados de nascimentos no HSS no período de estudo. Foram critérios de exclusão a ausência de registro de IG ou $\mathrm{PN}$, natimortalidade e IG $>43$ semanas ou $<24$ semanas, sendo o último critério justificado por não permitir análise pela curva Lubchenco et al. 4

Variáveis do estudo: sexo, PN, IG - categorizada em $<32$ semanas, 32 a 36 semanas completas, 37 a 39 semanas completas e $\geq 40$ semanas - e PIG considerando o PN inferior ao percentil 10 para a IG, de acordo com as curvas Lubchenco et al. 4 e Fenton e Kim. ${ }^{8}$ A escolha dos dois referenciais deveu-se aos seguintes fatos: 1) o serviço materno-infantil do HSS ainda adota as curvas de Lubchenco et al. ${ }^{4}$ para todos os RN como referencial de PN para a IG e pode estar subestimando o número de PIG que deveriam estar sendo monitorados para hipoglicemia quando se compara com o uso de curvas mais atuais construídas a partir de maior número de recémnascidos para cada idade gestacional como as curvas de Fenton e Kim. ${ }^{8}$

A análise descritiva foi apresentada em frequências, números absolutos e medidas de tendência central (medianas e valores máximos e mínimos após aplicar o teste Kolmogorov-Smirnov para testar a normalidade da distribuição das variáveis $\mathrm{PN}$ e $\mathrm{IG)}$.

O diagnóstico de PIG foi comparado segundo as duas curvas, Lubchenco et al. ${ }^{4}$ e Fenton e Kim. ${ }^{8}$ Também as variáveis IG e sexo foram comparadas quanto ao diagnóstico de PIG segundo as curvas. Para mensurar a concordância entre as curvas de Lubchenco et al. ${ }^{4}$ e Fenton e Kim, 8 utilizou-se a 
estatística Kappa $(k)^{10}$ com intervalo de confiança de $95 \%$. Sua interpretação realizou-se de acordo com critérios utilizados por Landis e Koch11: pobre (valores entre 0,00 e 0,20$)$, razoável $(0,21$ a 0,40$)$, moderada $(0,41$ a 0,60$)$, substancial $(0,61$ a 0,80$)$ e boa $(0,80$ e 1,00$)$.

Foram construídas curvas a partir dos percentis 10 de peso dos RN e referenciais Lubchenco et al. $4 \mathrm{e}$ Fenton e Kim, ${ }^{8}$ por IG e para ambos os sexos. Para isso, optou-se por ajustar uma função polinomial de terceiro grau que promove a suavização das curvas, suprimindo pequenas variações na sua forma e, então, permitindo melhor visualização. Polinômios são funções matemáticas contínuas e suaves em toda a reta, o que implica não haver no gráfico quebras ou saltos. Os polinômios do terceiro grau têm um ponto de inflexão, crescem sem limite à medida que os valores de x crescem e decrescem sem limite à medida que os valores de $\mathrm{x}$ diminuem, comportandose no infinito como uma reta. 12

Para análise de comparação entre as variáveis sexo, IG e PIG utilizou-se o teste do qui-quadrado de Pearson e, em caso de valor de $p<0,20$, aplicou-se a regressão logística bivariada para medir as associações entre as variáveis utilizando o referencial Fenton e Kim ${ }^{8}$ e obtendo-se como medida de efeito a razão de chances (odds ratio).

Utilizaram-se os softwares Excel (versão 2010; Microsoft Office) e Statistical Package for the Social Sciences (SPSS) para Windows (versão 20.0, SPSS Inc., Chicago, IL) para a elaboração do banco de dados, codificação, digitação e análises dos dados. Para as curvas Fenton e Kim 8 também foi usada a planilha Fenton growth chart calculations.13 Considerou-se significante o valor de $p<0,05$.

O estudo foi aprovado pelo Comitê de Ética em Pesquisa da UFV, com isenção de obtenção do termo de consentimento e é parte integrante de tese de doutorado em Ciência da Nutrição, desenvolvida no Departamento de Nutrição e Saúde da UFV.

\section{Resultados}

No período estudado, ocorreram no HSS 5067 nascimentos. Foram excluídos 2648 por indefinição da idade gestacional e 55 por natimortalidade. Após aplicação dos critérios de inclusão e exclusão, a população a ser avaliada constituiu-se de dados de 2364 recém-nascidos, sendo destes 300 pré-termos (12,7\%).

Na população estudada, as medianas de IG e PN foram, respectivamente, 39 semanas (24-42 semanas) e $3150 \mathrm{~g}(320-4565 \mathrm{~g})$. De acordo com a IG, os RN distribuíram-se da seguinte forma: $<32$ semanas, $2,7 \%(\mathrm{n}=64) ; 32$ a 36 semanas completas, $10,0 \%(n=236) ; 37$ a 39 semanas completas, $64,4 \%$ $(n=1522)$ e $>40$ semanas, $22,9 \%(n=542)$. Eram do sexo masculino $51,7 \%(\mathrm{n}=1222)$ e feminino $48,3 \%$ $(n=1142)$, sem diferenças em sua distribuição nos estratos de IG - dados não apresentados em tabela.

De acordo com a Tabela 1, observou-se que as curvas de Lubchenco et al. ${ }^{4}$ e Fenton e Kim ${ }^{8}$ apresentaram concordância razoável $(p<0,001 ; k=0,292)$ para identificar os PIG, com maior detecção destes pelas curvas de Fenton e $\mathrm{Kim}^{8}$ (diferença de 12,9 no valor percentual).

À medida que aumentou a IG houve tendência em redução no coeficiente Kappa. Evidenciou-se boa concordância entre as curvas de Lubchenco et al. 4 e Fenton e Kim 8 para os nascidos com menos de 32 semanas $(p<0,001 ; k=0,938)$. As curvas de Fenton e $\mathrm{Kim}^{8}$ detectaram mais PIG que as de Lubchenco et al. 4 a partir dessa IG, cuja concordância mostrou-se moderada para aqueles entre 32 e 36 semanas $(p<0,001 ; k=0,483$; diferença no valor percentual de $9,3)$, razoável no estrato entre 37 e 39 semanas $(p<0,001 ; k=0,267$; diferença de 11,9$)$, e pobre a partir das 40 semanas $(p<0,001 ; k=0,195$; diferença de 19,0).

Quanto ao sexo, as curvas de Fenton e Kim8 detectaram mais PIG, com concordância razoável para o sexo masculino ( $p<0,001 ; k=0,209$; diferença de 16,5 no valor percentual) e moderada para o feminino $(p<0,001 ; k=0,413$; diferença de 9,1$)$.

A Figura 1 demonstra as curvas construídas a partir dos percentis 10 de peso dos $\mathrm{RN}$ e dos referenciais Lubchenco et al. 4 e Fenton e Kim, ${ }^{8}$ para ambos os sexos. Visualmente, a curva do percentil 10 para o sexo masculino, especialmente após as 35 semanas de IG, tendeu a níveis inferiores aos do percentil 10 da curva de Fenton e Kim. ${ }^{8} \mathrm{O}$ sexo feminino não apresentou esse comportamento.

Pela estatística Kappa, demonstrou-se que as curvas de Fenton e $\mathrm{Kim}^{8}$ apresentaram uma concordância ruim entre os sexos $(k=0,17$; IC95\% $[0,009 ; 0,025])$ - dados não apresentados em tabela - o que demonstra o comportamento diferente das curvas dos percentis 10 de peso para o sexo masculino e feminino. O teste do qui-quadrado de Pearson confirmou que o nascimento PIG foi mais frequente no sexo masculino ( $p<0,001)$ (Tabela 2$)$. A análise de regressão bivariada comprovou a associação entre sexo masculino e nascimento PIG (OR= 1,59; IC95\%= 1,28-2,00; $p<0,001)$ pelo referencial de Fenton e Kim. ${ }^{8}$ 
Tabela 1

Número absoluto, porcentagem e coeficiente Kappa obtidos para o nascimento PIG, quanto às curvas de Lubchenco e Fenton - Hospital São Sebastião, Viçosa-MG, 01/01/2010 a 31/12/2012 ( $n=2364)$.

\begin{tabular}{|c|c|c|c|c|c|c|c|c|c|}
\hline \multirow[t]{3}{*}{ Variáveis } & \multicolumn{4}{|c|}{ Curvas Lubchenco et al.4 } & \multicolumn{4}{|c|}{ Curvas Fenton e Kim8 } & \multirow{3}{*}{$\begin{array}{c}\text { Kappa } \\
\text { IC95\% [inf.; sup.] }\end{array}$} \\
\hline & \multicolumn{2}{|c|}{ PIG } & \multicolumn{2}{|c|}{ Não-PIG } & \multicolumn{2}{|c|}{ PIG } & \multicolumn{2}{|c|}{ Não-PIG } & \\
\hline & $\mathrm{n}$ & $\%$ & $\mathrm{n}$ & $\%$ & $\mathrm{n}$ & $\%$ & $\mathrm{n}$ & $\%$ & \\
\hline Total & 77 & 3,3 & 2287 & 96,7 & 383 & 16,2 & 1981 & 83,8 & $0,292[0,242 ; 0,343]$ \\
\hline \multicolumn{10}{|l|}{ Sexo } \\
\hline Masculino & 33 & 2,7 & 1189 & 97,3 & 235 & 19,2 & 987 & 80,8 & $0,209[0,149 ; 0,268]$ \\
\hline Feminino & 44 & 3,9 & 1098 & 96,1 & 148 & 13,0 & 994 & 87,0 & $0,413[0,323 ; 0,495]$ \\
\hline \multicolumn{10}{|l|}{ IG (semanas) } \\
\hline$<32$ & 9 & 14,1 & 55 & 85,9 & 10 & 15,6 & 54 & 84,4 & $0,938[0,776 ; 1,000]$ \\
\hline $32-36,6$ & 12 & 5,1 & 224 & 94,9 & 34 & 14,4 & 202 & 85,6 & $0,483[0,285 ; 0,655]$ \\
\hline $37-39,6$ & 40 & 2,6 & 1482 & 97,4 & 220 & 14,5 & 1302 & 85,5 & $0,267[0,197 ; 0,338]$ \\
\hline$\geq 40$ & 16 & 3,0 & 526 & 97,0 & 119 & 22,0 & 423 & 78,0 & $0,195[0,111 ; 0,283]$ \\
\hline
\end{tabular}

PIG = pequeno para idade gestacional; IG= idade gestacional; IC95\%: intervalo de confiança; inf.= limite inferior; sup.= limite superior; $p<0,001$.

\section{Figura 1}

Curvas de percentis 10 (P10) de peso ao nascimento da população estudada e das curvas de Lubchenco et al.4 e Fenton e Kim8 de acordo com idade gestacional ao nascimento, para o sexo masculino (A) e feminino (B).
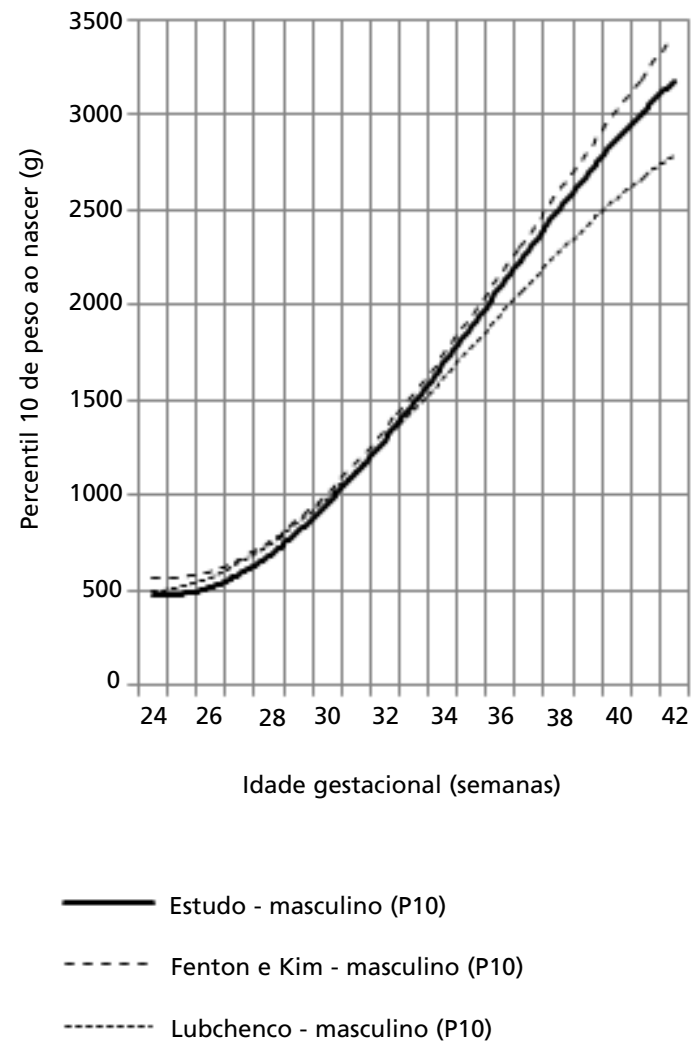
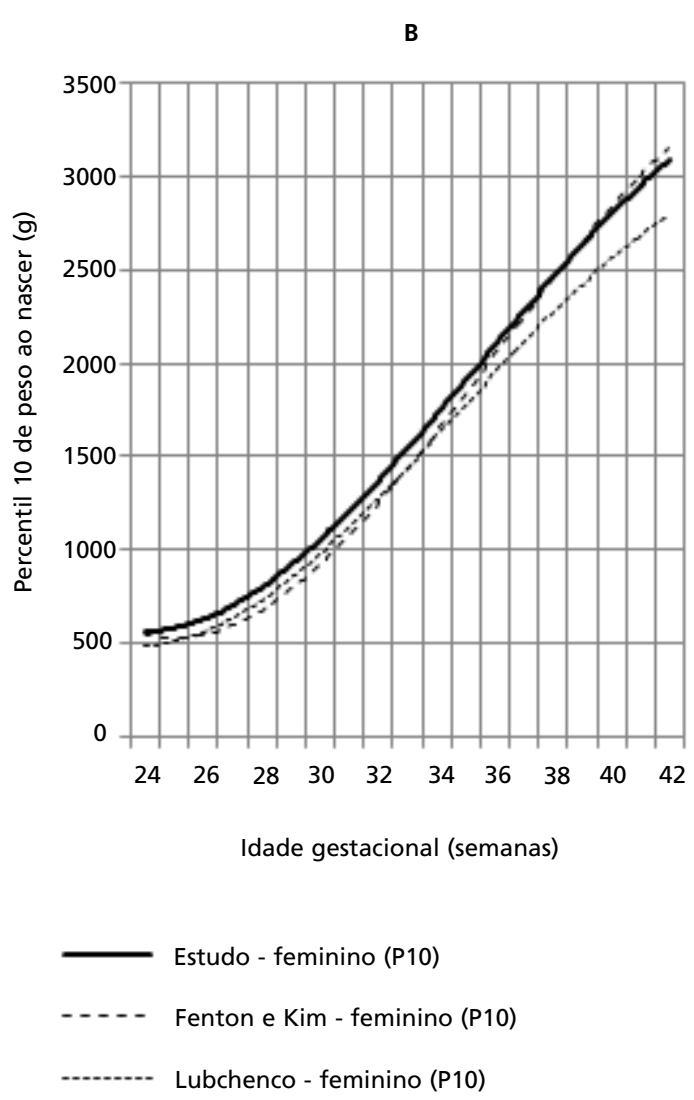
Análise de regressão bivariada de acordo com o nascimento PIG pelas curvas de Fenton e Kim 8 - Hospital São Sebastião, Viçosa-MG, 01/01/2010 a 31/12/2012 ( $n=2364)$.

\begin{tabular}{|c|c|c|c|c|c|c|c|}
\hline \multirow[t]{3}{*}{ Variáveis } & \multicolumn{4}{|c|}{ PIG (Fenton e Kim)8 } & \multirow[t]{3}{*}{$p^{*}$} & \multirow{3}{*}{$\begin{array}{l}\text { Odds ratio } \\
\text { (IC95\%) }\end{array}$} & \multirow[t]{3}{*}{$p^{* *}$} \\
\hline & \multicolumn{2}{|c|}{ Sim } & \multicolumn{2}{|c|}{ Não } & & & \\
\hline & $\mathrm{n}$ & $\%$ & $\mathrm{n}$ & $\%$ & & & \\
\hline Sexo & & & & & $<0,001$ & - & - \\
\hline Masculino & 235 & 19,2 & 987 & 80,8 & - & $1,59(1,28-2,00)$ & $<0,001$ \\
\hline Feminino & 148 & 13,0 & 994 & 87,0 & - & - & - \\
\hline
\end{tabular}

PIG= pequeno para a idade gestacional; IC95\%: intervalo de confiança de $95 \%$; A variável idade gestacional apresentou valor $p>0,20$ e não foi incluída na análise de regressão logística; *Teste do qui-quadrado de Pearson; **Análise de regressão logística bivariada.

\section{Discussão}

O estudo evidenciou que as curvas de Lubchenco et al. 4 podem estar subestimando o número de recémnascidos PIG. As curvas de Fenton e Kim, 8 em comparação às de Lubchenco et al.,4 apresentaram maior capacidade de detecção de RN PIG. Em geral, nas unidades neonatais, curvas de distribuição de peso para idade gestacional são usadas para identificação de recém-nascidos em risco para o desenvolvimento de doenças metabólicas e muitas vezes como proxy de restrição de crescimento intrauterino. A escolha da curva a ser usada portanto deve recair sobre aquela com melhor possiblidade de detectar um grupo de risco para uma determinada situação de risco à saúde.

As diferenças na metodologia de construção das curvas, nas populações e épocas estudadas podem explicar as diferenças encontradas. As curvas de Fenton e Kim, ${ }^{8}$ desenvolvidas a partir de uma metanálise, usando populações de vários países e um grande número de recém-nascidos para cada idade gestacional têm se mostrado atualmente mais adequadas para o uso como referência do que as curvas de Lubchenco et al., ${ }^{4}$ que se basearam em uma única população de Denver, recém-nascidos da década de 1950 e início da década de 1960 predominantemente de imigrantes com baixo poder socioeconômico, fatores que podem ter influenciado o peso ao nascer trazendo a curva para baixo. ${ }^{4,8}$

Uma outra questão importante é destacar que a classificação de um RN PIG requer o conhecimento exato da IG, medição antropométrica precisa e pontos de corte baseados em uma população representativa.14,15 A curva de Lubchenco et al.,4 que utilizou a data da última menstruação para cálculo da IG, pode ter apresentado acurácia reduzida na obtenção dos dados. 14,15 Além disso, recomenda-se utilizar curvas específicas para os $\operatorname{sexos}^{14} \mathrm{e}$ construídas a partir de medidas antropométricas de crianças nascidas após 1990, com atualização periódica e considerando a tendência secular.16-18

As taxas de nascimento de PIG no presente estudo foram de $16,2 \%$ quando avaliadas pelas curvas de Fenton e Kim, 8 contrastando com apenas 3,3\% pelas curvas Lubchenco et al. 4 Considerando que aproximadamente $10 \%$ dos RN a termo são PIG em países desenvolvidos, comparados a taxas de até $23 \%$ naqueles em desenvolvimento, 19 no estudo, as taxas de RN PIG estariam subestimadas pelo uso das curvas de Lubchenco et al. ${ }^{4}$ Alexander et al. ${ }^{6}$ discutem este tópico e consideram que as curvas publicadas antes de 1990 estão desatualizadas para classificar os RN PIG e mencionam as influências das mudanças nas práticas nutricionais e crescimento populacional ao longo do tempo e da falta de representatividade de algumas curvas.

Para os RN com IG inferior a 32 semanas, a curva de Lubchenco et al. ${ }^{4}$ e Fenton e Kim ${ }^{8}$ foram concordantes. Contudo, o menor tamanho amostral nesse estrato pode ter influenciado tais resultados. A partir dessa IG, a discordância foi crescente entre as curvas, porém mantendo-se a maior capacidade de detecção dos PIG pelas curvas Fenton, fato de relevância clínica. De maneira semelhante, no estudo de Ayerza Casas et al.20 as curvas de Lubchenco et al. 4 detectaram menos PIG quando comparadas a 
outras. Os autores afirmam que a correta identificação dos RN PIG permite melhor avaliação dos seus riscos a curto e longo prazo. ${ }^{20}$

Tanto entre os prematuros quanto entre os termos há diversos problemas clínicos associados ao nascimento PIG, tais como hipoglicemia, asfixia perinatal, dificuldades na termorregulação, maior risco de mortalidade e prejuízos futuros no crescimento e desenvolvimento. Nascer PIG se associa à restrição do crescimento extrauterino e ao maior desenvolvimento de doenças crônicas na idade adulta diabetes, hipertensão e doença coronariana.3,21-23 Dessa forma, a maior capacidade em revelar o grupo de risco possibilita implementação de estratégias que visem melhorias no cuidado neonatal e prognóstico dos RN em longo prazo. 14,24,25

Neste estudo, observou-se que, quando utilizado o referencial de Fenton e Kim, ${ }^{8}$ o nascimento PIG foi mais frequente no sexo masculino, com chance 1,6 vezes maior em relação ao sexo feminino. Contudo, não foi possível esclarecer o porquê deste fato, que merece ser investigado. Goodman et al.26 relacionam o stress adaptativo materno a maiores taxas de nascimento PIG entre RN termos masculinos afro-americanos. Na literatura, são descritos como fatores associados ao desfecho PIG, sem distinção quanto ao sexo: renda familiar inferior a um salário mínimo, pré-natal de baixa qualidade, exposição ao tabaco e doença hipertensiva. 27,28

Rodrigues et al. 15 comparam as curvas de Fenton e $\mathrm{Kim}^{8}$ às de Alexander et al.6 e descrevem as primeiras como instrumento estatístico mais robusto

\section{Referências}

1. Gardosi J, Madurasinghe V, Williams M, Malik A, Francis A. Maternal and fetal risk factors for stillbirth: population based study. BMJ. 2013; 346: f108.

2. Barros FC, Huttly SR, Victora CG, Kirkwood BR, Vaughan JP. Comparison of the causes and consequences of prematurity and intrauterine growth retardation: a longitudinal study in southern Brazil. Pediatrics. 1992; 90 (2 Pt 1): 23844.

3. Barker DJ. The fetal and infant origins of adult disease. BMJ. 1990; 301 (6761): 1111.

4. Lubchenco LO, Hansman C, Dressler M, Boyd E. Intrauterine growth as estimated from live-born birth weight data at 24 to 42 weeks of gestation. Pediatrics. 1963; 32: 793-800.

5. Usher R, McLean F. Intrauterine growth of live-born Caucasian infants at sea level: standards obtained from measurements in 7 dimensions of infants born between 25 and 44 weeks of gestation. J Pediatr. 1969; 74 (6): 901-10.

6. Alexander GR, Himes JH, Kaufman RB, Mor J, Kogan M. no diagnóstico de RN PIG, construídas com informações mais recentes e que permitem a avaliação do crescimento por três parâmetros (estatura, perímetro cefálico) e por sexo.

Apesar dos vários estudos em busca de um referencial de crescimento intrauterino, ainda não há na literatura consenso sobre uma referência a ser usada. ${ }^{15}$ Mas, até o momento, as curvas de Fenton e $\mathrm{Kim}^{8}$ são as que mais se aproximam de uma referência adequada. Destacamos o ineditismo do presente trabalho, pois não encontramos na literatura brasileira outros autores que tenham comparado a detecção de PIG pelas curvas de Lubchenco et al. ${ }^{4} \mathrm{e}$ Fenton e Kim. ${ }^{8}$ Como limitações deste estudo, mencionamos sua característica transversal, sujeita a vieses de informações, e a ausência de dados pré e perinatais que poderiam elucidar a maior ocorrência de PIG entre os RN do sexo masculino. Pretendemos despertar o interesse para o desenvolvimento de mais estudos sob essa ótica e futuros estudos devem ser endereçados à capacidade de detecção de hipoglicemia dependendo da curva usada como referência para monitorização.

O uso do percentil 10 das curvas de Fenton e $\mathrm{Kim}^{8}$ como referencial possibilitou maior detecção dos RN PIG. O nascimento PIG foi mais frequente no sexo masculino, com chance 1,6 vezes maior em relação ao feminino. A maior revelação do grupo de risco contribui para adoção de estratégias visando melhorias no cuidado neonatal e prognóstico em longo prazo.

A United States national reference for fetal growth. Obstet Gynecol. 1996; 87 (2): 163-8.

7. Olsen IE, Groveman SA, Lawson ML, Clark RH, Zemel BS. New intrauterine growth curves based on United States data. Pediatrics. 2010; 125 (2): e214-24.

8. Fenton TR, Kim JH. A systematic review and meta-analysis to revise the Fenton growth chart for preterm infants. BMC Pediatr. 2013; 13: 59.

9. Villar J, Cheikh Ismail L, Victora CG, Ohuma EO, Bertino E, Altman DG, Lambert A, Papageorghiou AT, Carvalho M, Jaffer YA, Gravett MG, Purwar M, Frederick IO, Noble AJ, Pang R, Barros FC, Chumlea C, Bhutta ZA, SH K. International Fetal and Newborn Growth Consortium for the 21 st Century (INTERGROWTH-21st). International standards for newborn weight, length, and head circumference by gestational age and sex: the Newborn CrossSectional Study of the INTERGROWTH-21st Project. Lancet. 2014; 384 (9946): 857-68.

10. Fleiss JL, Cohen J, Everitt BS. Large sample standard 
errors of kappa and weighted kappa. Psychol Bull. 1969; 12 (1): 323-7.

11. Landis JR, Koch GG. The measurement of observer agreement for categorical data. Biometrics. 1977; 33 (1): 159-74

12. Hauspie RC. Mathematical models for the study of individual growth patterns. Rev Epidém et Santé Publ. 1989; 37: 461-76.

13. Fenton TR, Sauve RS. Using the LMS method to calculate $\mathrm{Z}$-scores for the Fenton preterm infant growth chart. Eur J Clin Nutr. 2007; 61 (12): 1380-5.

14. Clark RH, Olsen IE, Spitzer AR. Assessment of neonatal growth in prematurely born infants. Clin Perinatol. 2014; 41 (2): 295-307.

15. Rodrigues FP, Martinelli S, Bittar RE, Francisco RPV, Zugaib M. Comparação entre duas curvas de crescimento para o diagnóstico de recém-nascidos pequenos para a idade gestacional. Rev Bras Ginecol Obstet. 2015; 37 (2): 59-63.

16. Fenton TR, Nasser R, Eliasziw M, Kim JH, Bilan D, Sauve $R$. Validating the weight gain of preterm infants between the reference growth curve of the fetus and the term infant. BMC Pediatr. 2013; 13 (1): 92

17. Cardoso-Demartini AA, Bagatin AC, Silva RPGVC, Boguszewski MCS. Crescimento de crianças nascidas prematuras. Arq Bras Endocrinol Metabol. 2011; 55 (8): 534-40.

18. Bhatia J. Growth curves: how to best measure growth of the preterm infant. J Pediatr. 2013; 162 (3 Suppl): S2-6.

19. Onis M, Blossner M, Villar J. Levels and patterns of intrauterine growth retardation in developing countries. Eur J Clin Nutr. 1998; 52 (Suppl 1): S5-15.

20. Ayerza Casas A, Rodríguez Martínez G, Samper Villagrasa MP, Ventura Faci P. Nacer pequeño para la edad gestacional puede depender de la curva de crecimiento utilizada. Nutrición Hospitalaria. 2011; 26: 752-8.

Recebido em 22 de junho de 2015

Versão final apresentada em 14 de dezembro de 2015

Aprovado em 4 de janeiro de 2016
21. Lima PA, Carvalho M, Costa AC, Moreira ME. Variables associated with extra uterine growth restriction in very low birth weight infants. J Pediatr (Rio J). 2014; 90 (1): 22-7.

22. Dusick AM, Poindexter BB, Ehrenkranz RA, Lemons JA. Growth failure in the preterm infant: can we catch up? Semin Perinatol. 2003; 27 (4): 302-10.

23. Singhal A, Cole TJ, Fewtrell M, Kennedy K, Stephenson T, Elias-Jones A, Lucas A. Promotion of faster weight gain in infants born small for gestational age: is there an adverse effect on later blood pressure? Circulation. 2007; 115 (2): 213-20.

24. Doctor BA, O'Riordan MA, Kirchner HL, Shah D, Hack M. Perinatal correlates and neonatal outcomes of small for gestational age infants born at term gestation. Am J Obstet Gynecol. 2001; 185 (3): 652-9.

25. Malin GL, Morris RK, Riley R, Teune MJ, Khan KS. When is birthweight at term abnormally low? A systematic review and meta-analysis of the association and predictive ability of current birthweight standards for neonatal outcomes. BJOG. 2014; 121 (5): 515-26.

26. Goodman JM, Karasek D, Anderson E, Catalano RA. The contribution of attenuated selection in utero to small-forgestational-age (SGA) among term African American male infants. Soc Sci Med. 2013; 88: 83-9.

27. Zambonato AM, Pinheiro RT, Horta BL, Tomasi E. Risk factors for small-for-gestational age births among infants in Brazil. Rev Saúde Pública. 2004; 38 (1): 24-9.

28. Franciotti DL, Mayer GN, Cancelier ACL. Fatores de risco para baixo peso ao nascer: um estudo de caso-controle. Arq Cat Med. 2010; 39 (3): 63-9. 